REZENSION

\section{Transdisziplinär und transformativ forschen}

\author{
Eine Methodensammlung
}

Marianne Penker, Institut für nachhaltige Wirtschaftsentwicklung, Feistmantelstr.4, 1180 Wien (penker@boku.ac.at),orcid.org/0000-0002-5185-9558

Basierend auf forschungspraktischen Erfahrungen in Reallaboren in Baden-Württemberg, präsentiert dieses Open-AccessBuch Methoden für transdisziplinäre und transformative Forschung. Diese Methoden unterstützen die gemeinsame Generierung und Synthese von Wissen durch Wissenschaft und Praxis und zwar für transformative wie nicht-transformative Forschung, für solche innerhalb wie außerhalb von Reallaboren. Die Methodensammlung ergänzt eine zunehmende Zahl handbuchartiger Werke und Online-Sammlungen, in denen im weitesten Sinne transdisziplinäre Methoden sowie Dialog-Methoden vorgestellt werden (S. 45 bzw. S. 47). Das Besondere dieser Sammlung ist sicher der Fokus auf transformative Forschung in Reallaboren sowie der Versuch, die Methoden entlang desselben Rasters von zuvor definierten Kriterien für Methodenwahl, -umsetzung und -reflexion darzustellen.

\section{Teil 1:}

\section{Die vorangestellten Reflexionen}

Den detaillierten Methodenbeschreibungen vorangestellt sind Reflexionen über transdisziplinäres und transformatives Forschen. Im ersten Kapitel wird basierend auf einer Literaturanalyse die Struktur erarbeitet, die der Methodensammlung im zweiten Kapitel zugrunde liegt. Es werden Kriterien für eine am Kontext und Ziel orientierte Methodenwahl, für eine effektive, transparente, ethische, theorie-, gegenstands- und akteursbezogene Methodenumsetzung und -kombination sowie für eine auf die (wahrgenommenen) Wirkungen bezogene Methodenreflexion definiert (S. 54 ff.). In weiteren Kapiteln werden zentrale Begriffe und Akteure, die Ziele und Designprinzipien von Reallaboren sowie Leitfragen, Qualitätskriterien und Mythen der Partizipation diskutiert.

This is an article distributed under the terms of the Creative Commons Attribution License CCBY 4.0 (https://creativecommons.org/licenses/by/4.0/)

https://doi.org/10.14512/tatup.28.1.79
Teil 2:

\section{Die Methodensammlung}

Der zweite und wohl auch Hauptteil des Buches widmet sich der Beschreibung von sechs bereits praktisch erprobten Methodenpaketen. Diese unterstützen einzelne Phasen, teilweise aber auch den gesamten Prozess: von partizipativer Themenfindung, über Wissenserzeugung und Wissensintegration bis hin zur konkreten Intervention bzw. Transformation und deren Reflexion. Alle sechs Methodenkapitel orientieren sich mehr oder weniger an der gemeinsamen Struktur der zuvor definierten Gütekriterien. Sie zeigen anhand einer Schritt-für-Schritt-Anleitung und mit Hilfe von konkreten Beispielen, wie sich durch die Kombination von aufeinander abgestimmten Methoden unterschiedliche Praxis-, Forschungs- und Bildungsziele erreichen lassen. Die Methoden sind ausführlich beschrieben und mit Beispielen illustriert.

Die „Wissensmesse“ ist ein mehrstufiger, partizipativer und wettbewerbsbasierter Abstimmungsprozess zwischen Wissenschaft und Praxis. Er zielt auf die Generierung eines Forschungsprogramms ab, das sowohl den Ansprüchen der Forschung als auch der Praxis gerecht wird. Das zweite Methodenkapitel zeigt Schritt für Schritt den Weg zum Realexperiment und bündelt Methoden, um Schlüsselakteure zu identifizieren, Kooperationsstrukturen aufzubauen und seitens der Zivilgesellschaft entwickelte Projektideen auszuwählen, die dann in Realexperimenten umgesetzt werden. Das dritte Methodenkapitel zeigt, wie Realexperimente initiiert, begleitet und beforscht werden können, um mit einer Reihe von solchen Experimenten Impulse für soziale Innovationen zu setzen. „Zwischen Wunsch und Wirklichkeit" - so der Titel des vierten Methodenkapitels - verspricht eine Anleitung für einen transdisziplinären Visionen-Workshop mit der Bevölkerung, der mit Methoden der Modellierung und Visualisierung kombiniert wird. Ihm schließt sich eine Wissensbilanzierung in transdisziplinären Netzwerken an, welche sich in der Regel durch eine geringe Verfasstheit auszeichnen. Durch die Kombination unterschiedlicher Erhebungsmethoden sollen Human-, Struktur- und Beziehungskapital systematisch analysierbar und sichtbar gemacht werden. Die Methodensammlung schließt mit einer transdisziplinären und transformativen $\mathrm{Me}$ thode für die universitäre Lehre. „Wissen to Go“ zeigt, wie Stu-

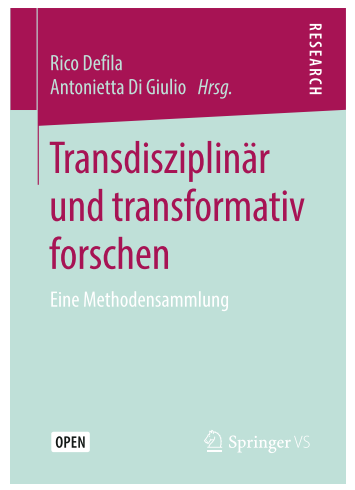

Defila, Rico; Di Giulio, Antonietta (Hg.) (2018): Transdisziplinär und transformativ forschen. Eine Methodensammlung. Wiesbaden: Springer VS, 406 S., open access eBook, ISBN 9783658215309 , https://doi.org/10.1007/978-3-658-21530-9 
dierende gemeinsam mit (außer-)universitären AkteurInnen Forschungsdesigns konzipieren, praktisch in Reallaboren umsetzen und in Hinblick auf Lerneffekte reflektieren können.

\section{Relevanz für eine transdisziplinäre Methodologie}

Auch wenn die vorgestellten Reflexionen und Methoden aus der forschungspraktischen Erfahrung in Reallaboren gewonnen wurden, adressieren sie sehr wohl grundlegende Fragen transdisziplinärer Forschung, die weit über das Forschungsformat Reallabor oder die transformative Nachhaltigkeitsforschung hinausgehen. Die Methodensammlung soll - so die Herausgeber - auch anderen gesellschaftspolitisch und ethisch legitimierten Zielen mit großer gesellschaftlicher Relevanz dienen. Neben Gesundheit und Sicherheit könnte so auch die Technikfolgenabschätzung ein interessantes Anwendungsfeld darstellen, da die Methoden entlang einer einheitlichen, vom Forschungsgegenstand weitgehend losgelösten Struktur so detailliert präsentiert werden, dass auch Dritte damit ein wertvolles methodisches Rüstzeug an die Hand bekommen.

Dieses Buch leistet einen wesentlichen Beitrag für eine informierte und auf die jeweiligen Bedingungen abgestimmte Methodenwahl sowie für einen reflektierten Methodeneinsatz, der auf die Qualität von transdisziplinären Prozessen und Ergebnissen abzielt. Auch wenn ein abschließendes Kapitel fehlt, das die vorgestellten Methoden vergleichend gegenüberstellt, haben die in diesem Buch erarbeiteten Kriterien großes Potenzial für eine solche Methodenreflexion (wie z. B. Stärken/Schwächen der Methoden bezüglich Einsatzgebiet oder Zeitpunkt, Theorieund Wertgeladenheit der Methode, Machtstrukturen und Implikationen für den Methodeneinsatz, Balance zwischen reflexiver Distanz und Involvierung, Erwartungsmanagement). Es ist

\section{Dieses Buch leistet einen wesent-} lichen Beitrag für einen reflektierten Methodeneinsatz, der auf die Qualität von transdisziplinären Prozessen abzielt.

zu hoffen, dass diese Kriterien - weit über die vorliegende Methodensammlung hinaus - einen vertieften und kritischen Austausch über Methodenwahl und -einsatz strukturieren und so zu einer transdisziplinären Methodologie beitragen.

\section{GAIA Miaters Student Paper Award}

The international journal GAIA - Ecological Perspectives for Science and Society invites liasters students to participate in the 2020 GAIA liasters Student Paper Award.

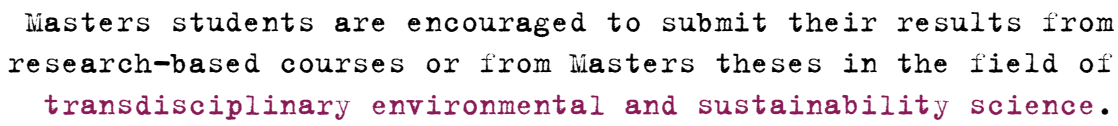

The winner will be selected by an international jury and will be granted a prize money of EUR 1,500 endowed by the Selbach Umwelt Stiftung and Dialogik gGmbH, as well as a free one-year subscription to GAIA, including free online access. The winner may also be invited to submit his or her paper for publication in GAIA. 